EPJ Web of Conferences 73, 07007 (2014)

DOI: $10.1051 /$ epjconf/20147307007

(C) Owned by the authors, published by EDP Sciences, 2014

\title{
Neutron skin studies of medium and heavy nuclei
}

\author{
M. Thiel ${ }^{1, \mathrm{a}}$, D. Becker ${ }^{1}$, M. Ferretti ${ }^{1}$, K. Kumar ${ }^{2}$ and C. Sfienti ${ }^{1}$ \\ ${ }^{1}$ Institut für Kernphysik, Johannes Gutenberg-Universität Mainz, Germany \\ ${ }^{2}$ Department of Physics, University of Massachusetts Amherst, MA 01003-9337, USA
}

\begin{abstract}
The recent PREX experiment at JLab has demonstrated the sensitivity of parity violating electron scattering to the neutron density, meanwhile outlining its major experimental challenges. On the other side, intermediate energy photons are an ideal probe for studying the properties of strongly interacting matter from the nuclear scale down to the sub-nuclear components of the nucleus. Among others coherent pion photoproduction can provide information on the existence and nature of neutron skins in nuclei. The simultaneous combination of different techniques allows a systematic determination across the periodic table thus benchmarking modern calculation. Recently a systematic investigation of the latter method has been exploited at MAMI (Mainz). At MESA the same setup as in the measurement of the weak mixing angle can be used to determine the parity-violating asymmetry for polarized electrons scattered on heavy nuclei with a $1 \%$ resolution. Status and prospects of the projects are presented.
\end{abstract}

\section{Equation of state: From the lab to the stars}

One of the most fundamental observables of nuclei are the neutron and proton density distributions. Concerning symmetric nuclear matter both density distributions and the corresponding radii are similar, although the accuracy of the experimentally determined neutron radii is poor compared to the one of the proton radii. Going to neutron rich matter the excess neutrons are pushed out against the surface tension and the developed difference between the neutron and proton root-mean-square radii is called a neutron skin $\Delta R_{n p}$. Here, the thickness of the skin is directly related to the pressure. Yet, it is the same pressure that supports a neutron star against gravity. Even though heavy nuclei and neutron stars are orders of magnitude in size and weight apart, both quantities are sensitive to the nuclear equation of state (EOS)

$$
E(\rho, \delta)=E(\rho, 0)+S(\rho) \delta^{2}
$$

with the relative asymmetry parameter $\delta=\left(\rho_{n}-\rho_{p}\right) /\left(\rho_{n}+\rho_{p}\right)$. In particular, the neutron skin thickness of heavy nuclei is strongly correlated to the slope of the symmetry energy $S(\rho)$ at saturation density [1]. Hence, an accurate experimental determination of the neutron skin thickness of heavy nuclei would provide considerable constraints on the density dependence of the nuclear symmetry energy, in addition of being of substantial importance by itself.

\footnotetext{
ae-mail: thiel@kph.uni-mainz.de
}

This is an Open Access article distributed under the terms of the Creative Commons Attribution License 4.0, which permits unrestricted use, distribution, and reproduction in any medium, provided the original work is properly cited. 

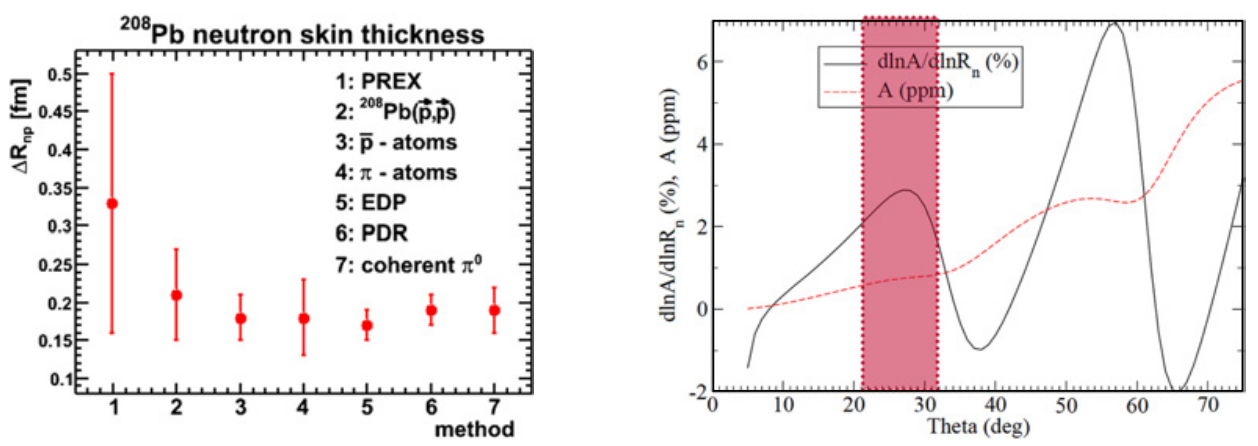

Figure 1. Left: neutron skin thickness $\Delta R_{n p}$ of ${ }^{208} \mathrm{~Pb}$ determined with various methods. Right: sensitivity of the parity violating asymmetry $A_{P V}$ for ${ }^{208} \mathrm{~Pb}$ to changes in the neutron radius $R_{n}$ as a function of the scattering angle $\theta$ for a beam energy of $200 \mathrm{MeV}$ (solid black line). The dashed red line represents the asymmetry $A_{P V}$. The red marked area is of special interest for future parity violating electron scattering experiments at MESA.

\section{The neutron skin story}

In recent years, the determination of neutron skin thickness has attracted considerable attention. Focussing on ${ }^{208} \mathrm{~Pb}$ nuclei, various methods have been used to extract this quantity: hadron scattering experiments (involving protons [2], antiprotons [3] and pions [4]), electric dipole polarizabilities [5], pygmy dipole resonances [6], coherent $\pi^{0}$ photoproduction [7], and parity violating electron scattering [8]. All methods except the latter one have to rely on model calculations to extract information on the neutron radius $R_{n}$. Hence, it is astonishing and impressive that all results agree within their error bars (Fig. 1, left panel). However, the main goal, of course, is a direct measurement of $R_{n}$ which was first achieved by the ${ }^{208} \mathrm{~Pb}$ Radius EXperiment at Jefferson Laboratory. In the following, coherent $\pi^{0}$ photoproduction and parity violating electron scattering experiments will be introduced in detail.

\subsection{Coherent $\pi^{0}$ photoproduction at MAMI}

The method of coherent $\pi^{0}$ photoproduction $\left(\gamma+A_{\text {g.s. }} \rightarrow \pi^{0}+A_{\text {g.s. }}\right)$ provides an efficient tool to study the neutron skin of various nuclei. Here, the initial state is almost ideal: the photon probes, without any Coulomb scattering effects, the whole volume of the nucleus. Since in the incident photon energy range of interest (from threshold to $350 \mathrm{MeV}$ ) protons and neutrons contribute with the same amplitude to the coherent photoproduction of the $\pi^{0}$, the reaction is sensitive to the distribution of nucleons. Therefore, the measured cross section is proportional to the nuclear mass form factor times the mass number squared:

$$
\frac{d \sigma}{d \Omega}(\mathrm{PWIA})=\frac{s}{m_{N}^{2}} \times \frac{1}{2} \frac{q_{\pi}^{*}}{k^{*}}\left|F_{2}\left(E_{\gamma}^{*}, \theta_{\pi}^{*}\right)\right|^{2} \sin ^{2}\left(\theta_{\pi}^{*}\right) \times A^{2} F^{2}(q) .
$$

Information about the neutron skin thickness can be extracted by comparing the diffraction pattern of the measured cross section with theoretical calculations. Because the cross section is affected by final state interactions of the pion on the way out of the nucleus, this effect has to be accounted for in the model calculations, using a complex optical potential [9]. To describe the nucleon density within the theoretical calculation, a single symmetrised two parameter - half-height radius and diffuseness - Fermi distribution is used.

Already in 2005, a measurement of the coherent $\pi^{0}$ photoproduction on ${ }^{208} \mathrm{~Pb}$ has been performed at the Mainz Microtron (MAMI). Here, a neutron skin thickness of $\Delta R_{n p}=0.19 \pm 0.03($ stat $)+0.00-$ $0.03(s y s)$ fm could be determined [7]. In a novel campaign, the experimental program has been extended 
to measure in total four spin zero nuclei: ${ }^{58} \mathrm{Ni},{ }^{116} \mathrm{Sn},{ }^{120} \mathrm{Sn}$, and ${ }^{124} \mathrm{Sn}$. The latter ones are of special interest since they allow the determination of the neutron skin along an isotopic chain. Here the advantage is, that any systematic error due to pion-nucleon interaction will largely cancel. In addition, new data on ${ }^{208} \mathrm{~Pb}$ has been collected to cross check the results with the previous measurement. The photon beam was produced via Bremsstrahlung process by the electron beam impinging on a $10 \mu \mathrm{m}$ thin nickel foil. The scattered electrons were deflected in the magnetic field of a wide-range momentumdispersive electron spectrometer (Glasgow Photon Tagging Spectrometer [10]) and focussed onto the focal plane consisting of 353 plastic scintillators. They covered an energy range of appr. 5\% to $93 \%$ of the incident electron beam energy of $885 \mathrm{MeV}$ with an energy resolution of $\approx 2 \mathrm{MeV}$. Given the energies of the incoming and scattered electron, the energy of the photon is determined by $E=E_{0}-E_{e-}$. The detector system consists of the two calorimeter TAPS [11] and Crystall Ball [12], with the target located in the center of the latter detector. Covering almost the full solid angle, the system is perfectly suited to detect multi-photon final states. The detector system is completed by PID, Veto and MWPC for charged particle identification. The latter one was also used to determine the exact target position. The $\pi^{0}$ in the final state is detected via its two photon decay and its invariant mass is reconstructed in the offline analysis. Coherent and incoherent events can be separated using a missing energy analysis. The careful calibration and analysis of the data are currently ongoing.

\subsection{Parity violating electron scattering at MESA}

The future accelerator MESA (Mainz Energy recovering Superconducting Accelerator) will open the floodgates for a new generation of high precision parity violating electron scattering experiments in Mainz. With one experimental setup the weak mixing angle $\theta_{W}$ as well as the neutron radius $R_{n}$ will be measured. For the latter one highly polarized electrons (up to $P=85 \%$, intensities up to $I=150 \mu \mathrm{A}$ ) with maximum incident beam energy of $\mathrm{E}=200 \mathrm{MeV}$ will be scattered off a ${ }^{208} \mathrm{~Pb}$ target. In particular, the full azimuthal coverage of the detector will help to reduce the runtime of such experiments significantly.

Since the weak charge of the neutron is a factor 50 larger than the weak charge of the proton, a measurement of the parity violating asymmetry $A_{P V}$ is sensitive to the neutron density distribution:

$$
A_{P V}:=\frac{\sigma_{R}-\sigma_{L}}{\sigma_{R}+\sigma_{L}} \propto \frac{F_{w}\left(Q^{2}\right)}{F_{c h}\left(Q^{2}\right)}
$$

with the fourier transform of the weak charge density $F_{w}\left(Q^{2}\right)$ and the charge density $F_{c h}\left(Q^{2}\right)$. The related sensitivity of the parity violating asymmetry $A_{P V}$ to changes in the neutron radius $R_{n}$ is

$$
\varepsilon=\frac{d \ln \left(A_{P V}\right)}{d \ln \left(R_{n}\right)}=\frac{R_{n}}{A_{P V}} \frac{\delta A_{P V}}{\delta R_{n}} .
$$

Figure 1 (right panel) illustrates the sensitivity $\varepsilon$ versus the scattering angle $\theta[13]$. For ${ }^{208} \mathrm{~Pb}$ a peak sensitivity can be expected in a polar angular range between $21^{\circ}$ and $32^{\circ}$. Within this angular range, calculations have been performed - using two different settings with $\Delta \theta=2^{\circ}$ and $\Delta \theta=4^{\circ}$ - to verify the feasibility of such an experiment. Figure 2 (left panel) illustrates that a sensitivity of $0.5 \%$ for $\frac{\delta R_{n}}{R_{n}}$ can be reached within a reasonable running time, assuming a systematic uncertainty of the asymmetry of $1 \%$. For these kind of measurements it is essential, to separate the ground state of ${ }^{208} \mathrm{~Pb}$ from its first excited state $(\Delta \mathrm{E} \approx 3 \mathrm{MeV})$. Therefore, the position of the target, with respect to the center of the solenoid, and the strength of its magnetic field have been varied successively within a first iteration of simulation. With the target placed at $\mathrm{z}=-1.25 \mathrm{~m}$ and the detector at $\mathrm{z}=2.5 \mathrm{~m}$ the required discrimination between ground and first excited state can be achieved (Fig. 2, middle panel). A first estimation of the expected rate in the detector system $\left(\theta=23^{\circ}-27^{\circ}\right)$ is shown in the right panel of Fig. 2. A more detailed analysis using GEANT4 is currently ongoing. 

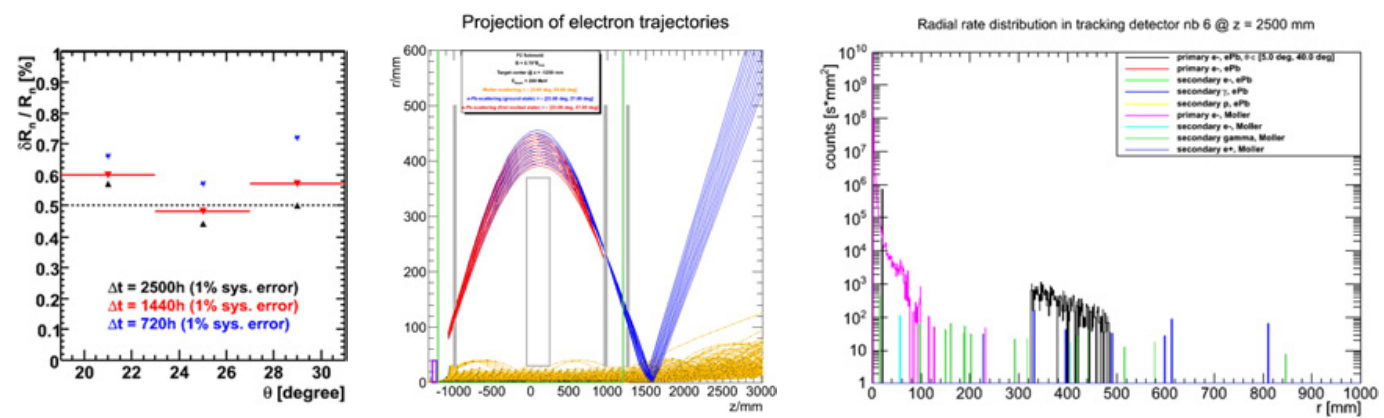

Figure 2. Left: estimation of running time for a $0.5 \%$ neutron radius determination. Middle: raytrace simulation to determine the best configuration. Electrons with $200 \mathrm{MeV}$ are shown in blue, electrons with $197 \mathrm{MeV}$ (belonging to the first excited state) in red and Møller electrons in yellow. The green lines represent the size of the solenoid whereas the grey lines show the lead shielding needed. Right: rate studies performed with GEANT4, taken the lead shieldings into account.

\section{Conclusions}

With the high quality electron beam accelerators MAMI and MESA an accurate determination of $R_{n}$ will be feasible. In particular, the interplay of coherent $\pi^{0}$ photoproduction and parity violating electron scattering experiments will help to provide novel constraints for nuclear- and astrophysics.

\section{References}

[1] X. Roca-Maza et al., Phys. Rev. Lett. 106, 252501 (2011)

[2] J. Zenihiro et al., Phys. Rev. C 82, 044611 (2010)

[3] B. Klos et al., Phys. Rev. C 76, 014311 (2007)

[4] C. Garcia-Recio et al., Nucl. Phys. A 547, 473 (1992)

[5] A. Tamii et al., Phys. Rev. Lett. 107, 062502 (2011)

[6] A. Carbone et al., Phys. Rev. C 81, 041301 (2010)

[7] D. Watts et al., submitted to Phys. Rev. Lett. (2013)

[8] S. Abrahamyan et al., Phys. Rev. Lett. 108, 112502 (2012)

[9] D. Drechsel et al., Nucl. Phys. A 660, 423 (1999)

[10] J.C. McGeorge et al., Eur. Phys. Jour. A 37, 129 (2008)

[11] A.R. Gabler et al., Nucl. Instr. Meth. A 346, 168 (1994)

[12] A. Starostin et al., Phys. Rev. C 64, 055205 (2001)

[13] S. Ban and C. Horowitz, private communication (2013) 